\title{
A Computer Simulation of Stress Transfer in Carbon
}

\section{Nanotube/Polymer Nanocomposites}

Jiawei Zhao and Mo Song*

Department of Materials, Loughborough University, Loughborough LE11 3TU, UK m.song@1boro.ac.uk

\section{Abstract}

The reinforcing efficiency or stress transfer of carbon nanotubes (CNT) on polymers in polymer/CNT composites mainly is controlled by the polymer-CNT interface. Enhancement of polymer-CNT interactions and interfacial crystallisation is as an important way for improvement of the reinforcement experimentally. However, it is not clear about the crystallisation and orientation of polymer chains on the CNT surface, and how the interfacial crystallisation layer affects the failure of the composite. In this work, poly(vinyl alcohol)/CNT nanocomposites was selected as an example and based on the molecular dynamics simulation, the crystallisation process, failure behaviour and stress transfer in poly(vinyl alcohol)/CNT nanocomposites were analysed. The crystallisation temperature of the polymer chains on the CNT surface is slightly higher than the bulk crystallisation temperature. CNT induced crystallisation can be divided into three stages: chain folding, orientating and growing on the CNT surface. A slower crack growth was observed in the interfacial crystallised polymer/CNT systems, compare to relative amorphous systems. The effect of the interfacial crystalline layer on stress transfer is similar as enhanced polymer-CNT interaction systems. The change of the polymer-CNT surficial energy to strain has been used to analyse the interfacial failure and the stress transfer. 


\section{Introduction}

Carbon nanotubes (CNTs) have generated a great deal of interest since their discovery, due to their unique physical properties. CNTs are known to have an extremely high Young's modulus of up to 1TPa and tensile strength approaching 60GPa. Much more research has focused on nanotubes as fillers in polymers with enhanced mechanical [1], thermal[2] and electrical properties [3]. Most significantly, CNTs are ideal candidates for the mechanical reinforcement of polymers. Although a templating transition model was used to explain ideal combination of polymers and nanotube diameters to maximally enhance the mechanical properties of composite structures, the disagreement between experimental data and theoretical values is obvious [4]. Significant mismatch of CNTs with polymer matrix exists, which cause the stress transform less effective. In order to reduce the mismatch and improve the stress transfer between CNTs and the polymer matrix, the researchers have focused on the interfacial interaction chemically [5-8] or interfacial crystallisation, such as in CNT/poly (vinyl alcohol) (PVA)[9-13], CNT/polyimide (PI) [14,15], and CNT/polyolefin[16]. An increment of the mechanical properties of polymers was reported when the interfacial crystallisation was formed in the CNT/PVA [9][12] [13], CNT/PI [14], CNT/PA[17]. Most of them were approached through anticipation method. On the other hand, the hybrid shish-kabab structure was also studied. The induced crystal was growth aligned with the longitudinal direction of reinforcement forming an hybrid Shish-kabab crystal[10,16-20] was reported, specifically the chain extension direction aligned with the longitudinal direction of Zigzag CNT.[18] Although limited information and 
reasons were given in explanation on the reinforcing effect of interfacial crystallisation in molecular level and the correlation behind the stress transfer, failure mechanism and the crystallisation mechanism on the surface of CNTs are still not clear enough due to the difficulties of observation experimentally. For observing configuration change in molecular level, the molecular dynamic (MD) is a useful tool. CNT related MD research can be commonly founded .[21,22] PVA has been widely investigated by MD with different scales, from DFT[23,24], full atom MD using reactive bond order potential [25] and pairwise potential [26] to coarse grain model [26-31] . Also, the mechanical behaviour [1] and crystallisation process [33] of CNT/PVA system was studied experimentally and could be used as validation for computer simulation. The study of load-transfer was based on the shear modulus of interfacial bonding between the continuous fibre and polymer matrix [34]. Little difference between amorphous and crystallised systems was reported. However, the method used in Ref [34] did not consider the interfacial fracture, which is commonly happened when the interfacial bonding was not strong enough. Another similar research conducted by MD simulation on coarse-grained PE/graphene/CNT hybrid composite indicates the crack propagation was affected by the topology of the carbon reinforcement.[35]. But the effect of crystallinity was not mentioned in the research. On the other hand, modelling work based on continuum phase modelling was developed[36-41] and applied to related nanocomposite system[42]. However, as restricted by modelling methodology, the detailed information about polymer/CNT interface was not clear. 
In this work, we will focus on 1) PVA crystallisation and configuration on CNT surface, 2) effect of formed crystalline layer on tensile mechanical behaviour and 3) stress transfer and a new analysis method based on computer simulation for analysis of the stress transfer. Reality, several factors might influence the interfacial behaviour, such as crosslink and functionalization. However, in our case, the effect of surface crystallisation on stress transfer and failure patent was being highlighted, so the polymer chains only physically interact with CNT.

\section{Model and methodology}

The polymer was simulated using coarse grain (CG) model, in which the polymer was abstracted into beads contains a single repeat unit, developed by Luo et al.[43]. CNT was described by AIREBO potential[21]. The potential which describes the behaviour between coarse grain poly(vinyl alcohol) (CGPVA) and CNT was conducted by iterative Boltzmann inversion method. Large-scale Atomic/Molecular Massively Parallel Simulator (LAMMPS) was used to simulate the system[44] and Ovito was used to visualised the system[45]. See supporting information for details.

For isothermal crystallisation, a relatively long time was needed for a MD simulation ( $\sim 300 \mathrm{~ns})$. To reduce the complexity of the simulation, the CNT was considered as a rigid body and the simulation was run in reduced unit same with Ref [43]. The CNT/PVA system was simulated in NpT ensemble, controlled by Nose-hover thermo and barostat [46]. The mixture was quenched down from $550 \mathrm{~K}$ to desired temperatures 
and hold for observation of the crystallisation.

For non-isothermal crystallisation, the CNT/PVA system was cooled from $550 \mathrm{~K}$ to $330 \mathrm{~K}$ at 1 bar with a cooling rate of $7.3 \times 10^{10} \mathrm{~K} / \mathrm{s}$, also controlled by Nose-Hoover thermostat and barostat.

Tensile test was carried out in NVT ensemble for two systems: short fibre and continuous fibre scenarios. The $\mathrm{Z}$ axis was elongated, and atoms remapped their position along $\mathrm{Z}$ axis during elongation during tensile test simulation. The elongation was controlled at a constant entering strain rate of $2.5 \times 10^{8} \mathrm{~s}^{-1}$. The temperature was controlled at $330 \mathrm{~K}$ by Nose-Hoover thermostat [46]. In the short fibre scenario, the simulation was conducted by cover all of the fibre with polymer beads and the load was transferred from the polymer to the CNT. In continuous fibre scenario, the CNT linked with itself through the periodic boundary and most of the load was generated by the elongation of the CNT.

\section{Result and discussion}

Isothermal crystallisation of the samples quenched was carried out from $451 \mathrm{~K}$ to $412.5 \mathrm{~K}$, respectively. The final configuration and the change of total energy with crystallisation time are shown in Fig. 1. 


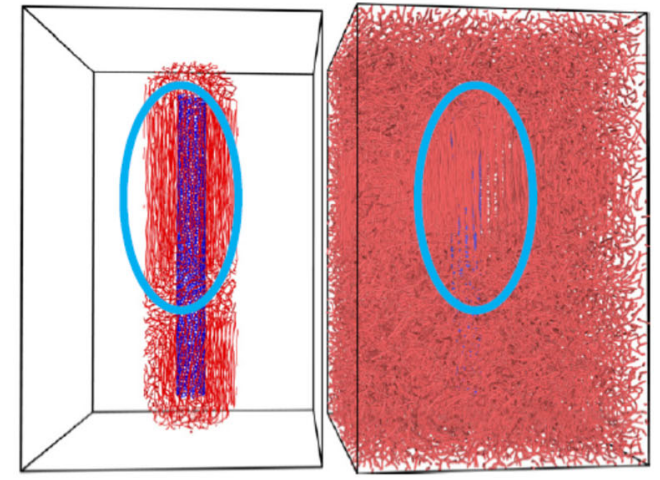

(a)
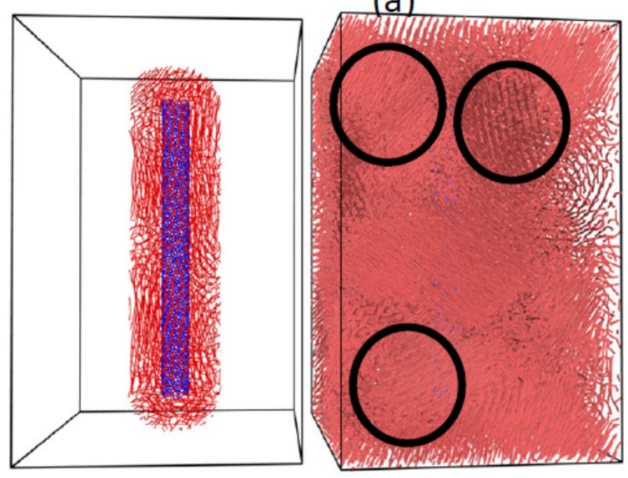

(c)
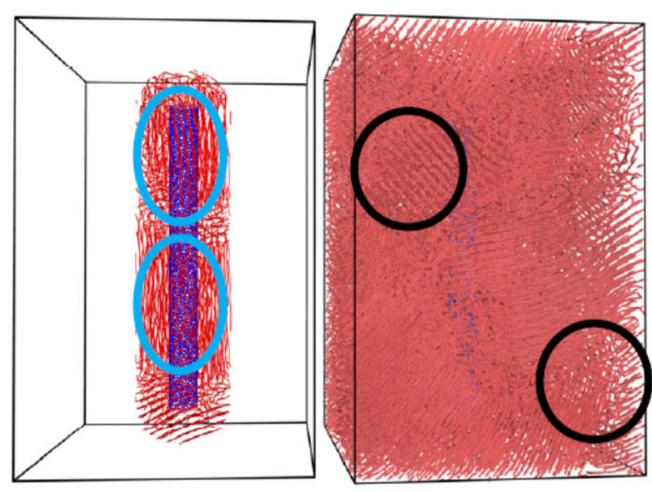

(b)

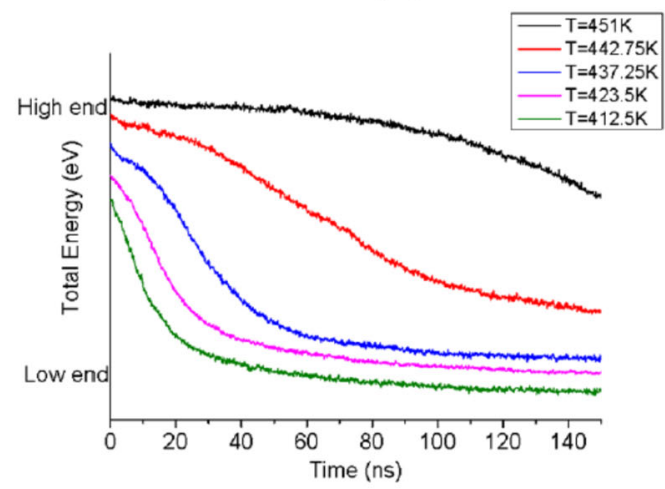

(d)

Randomly orientated polymeric crystal

Fig. 1. Final configuration and enthalpy change of isothermal crystallisation. (a) $\mathrm{T}=451 \mathrm{~K}$, (b) $\mathrm{T}=437.25 \mathrm{~K}$, (c) $\mathrm{T}=412.5 \mathrm{~K}$ and (d) Enthalpy against crystallisation time at different temperatures

The difference can be found from the energy dropping patents (Fig. 1(d)). For the CNT/CGPVA system crystallised at $437.25 \mathrm{~K}$ and $412.5 \mathrm{~K}$, the energy dropping patents are similar. But the energy dropping patent crystallised at $451 \mathrm{~K}$ shows a unique one which indicates the crystallisation process is different from that crystallised at $437.25 \mathrm{~K}$ and $412.5 \mathrm{~K}$. The energy dropping patent of $442.75 \mathrm{~K}$ is falling between $451 \mathrm{~K}$ and 437.25K, which indicates a smooth change took place when the crystallised temperature increased. 
Crystallisation at $451 \mathrm{~K}$ (Fig. 1(a)) produced only one kind of crystals growing from the CNT surface. Crystallisation at $437.25 \mathrm{~K}$ and $412.5 \mathrm{~K}$ (Fig. 1(b) and (c)) resulted in a considerable amount of random orientated crystals formed in the polymer matrix, which were not observed at the case of crystallisation at $451 \mathrm{~K}$. It is worth to mention that CNT-aligned polymeric crystals can also be formed in lower crystallisation temperatures. But the amount of formed crystals is considerable smaller than that crystallised at higher temperatures. At $451 \mathrm{~K}$, crystallisation in the polymer matrix did not take place. The CNT provides a possible nucleus, which cause the polymer crystals formed in CNT-aligned fashion. However, it seems the CNT-aligned crystals have a considerable slower growth rate. A possible reason is a stereo effect, i.e., CNT could block part of incoming melt chains to sustain crystal growth, and the polymer chain mobility could be restricted by the CNT.

Because the crystallisation in the polymer matrix cannot be formed at $451 \mathrm{~K}$ systems. $451 \mathrm{~K}$ was chosen to observation of crystallisation of the polymer on the CNT surface.

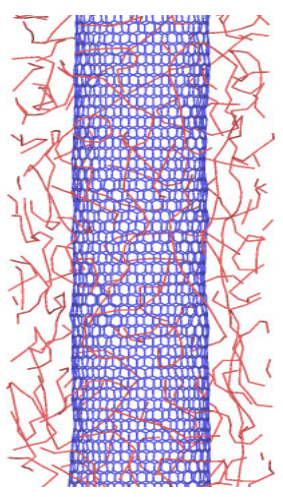

(a)

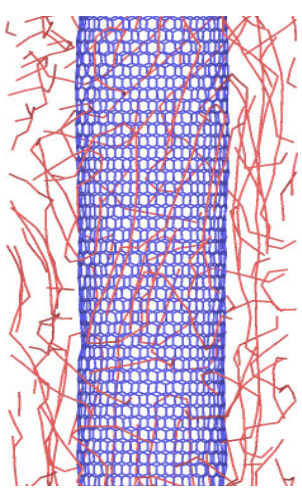

(b)

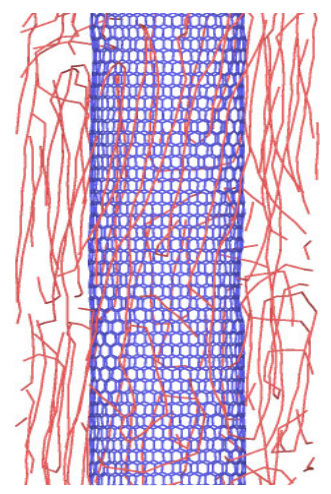

(c)

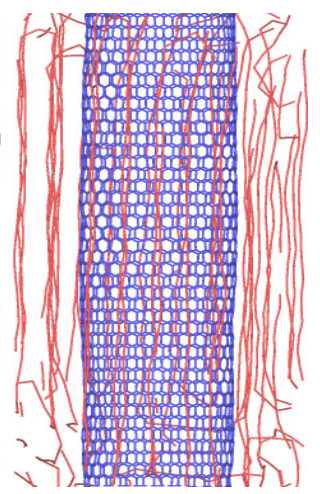

(d)

Fig. 2. Configuration of near-by polymer of a CNT during isothermal crystallisation at 
451K. (a) time $=0 \mathrm{~ns}$, (b) time $=0.75 \mathrm{~ns}$, (c) time $=2.25 \mathrm{~ns}$ and (d) time $=15 \mathrm{~ns}$

Above results indicate that CNT induced crystallisation can be divided into 3 stages: chain folding on the surface of CNT, orientating and growing. In the melt stage $(\mathrm{t}=$ 0ns), the nearby polymer was randomly distributed. At the initial stage of crystallisation, the polymer chains start to fold on the CNT surface shown in Fig. 2(b). The folding patent is quite disorientated. The angle between bonds of $37.8 \%$ polymer chains and CNT direction is about $15^{\circ}$ shown in Fig. 3.(a). The growth of the crystal on the CNT surface became harder as the length of the folded chain increased. An energy penalty must be paid for chain twist on the CNT surface or leave from the CNT surface if the growth direction remains.

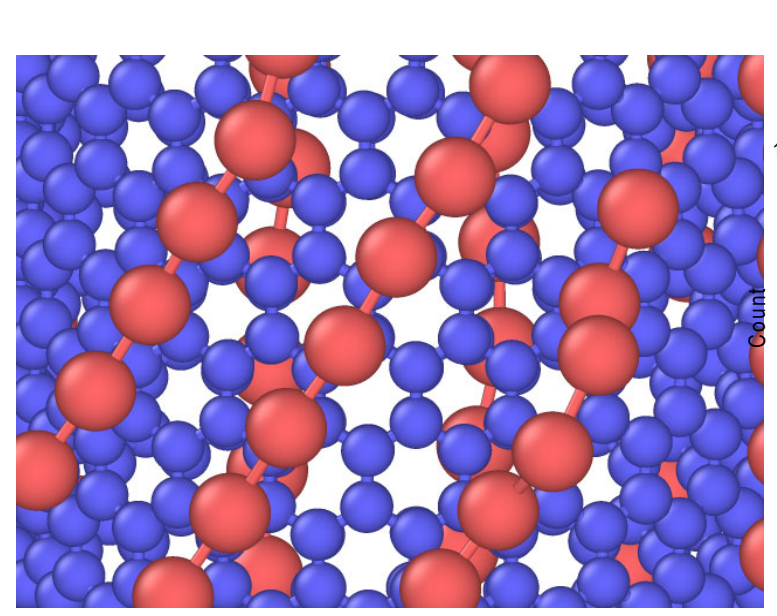

(a)

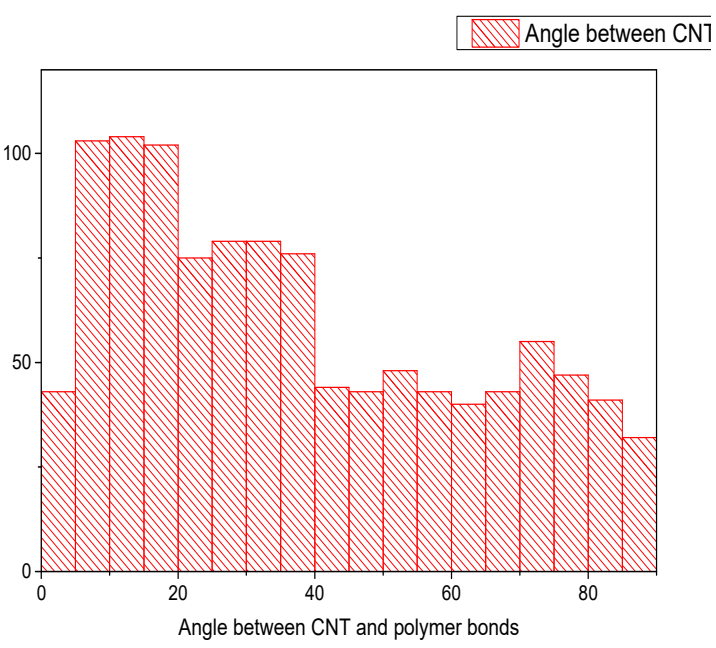

(b)

Fig. 3. Folding patent in the initial stage

After folding, the polymer chains start to align into CNT direction shown in Fig. 2(c).

From the state of Fig. 2(b) to that of Fig. 2(c), the folded chains with different angles start to compete each other and finally form a larger and more uniform structure. The 
change of configuration is shown in Fig. 4. In this stage, the energy favoured folded chain growth and others vanished. An ordered-absorbed layer of CGPVA was formed on the interface and the folding chains became orientated on CNT's direction. Some large aligned structures were also observed shown in Fig. 4 (d).

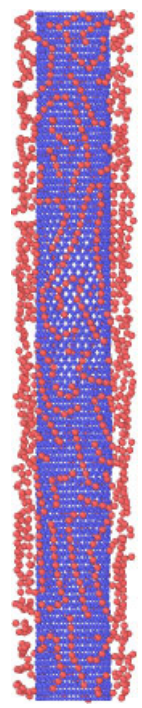

(a)

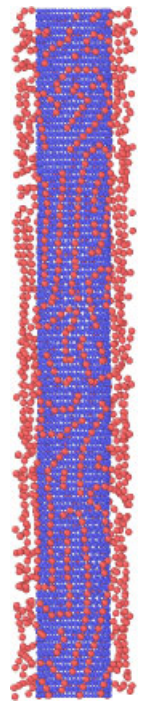

(b)

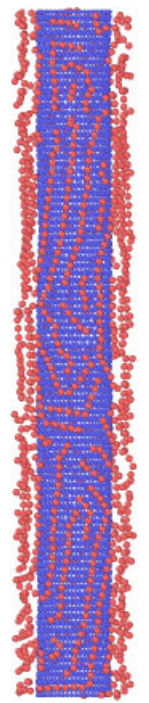

(c)

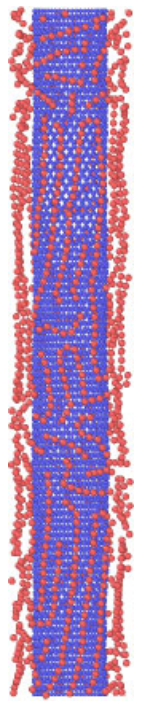

(d)

Fig. 4. Configuration refinement during isothermal crystallisation, (a) time $=0.75 \mathrm{~ns}$, (b) time $=1.5 \mathrm{~ns},(\mathrm{c})$ time $=2.25 \mathrm{~ns}$ and $(\mathrm{d})$ time $=3 \mathrm{~ns}$

At the growing stage, the orientated chains start to grow out of the interphase and finally the polymer crystal formed shown in Fig. 2(d), which is similar from previous observation from HR-TEM [10]. This can be confirmed from the observation of the conformation change of a single chain proxy to the CNT during crystal growth shown in Fig. 5. The part of chains near CNT were firstly crystallised. 


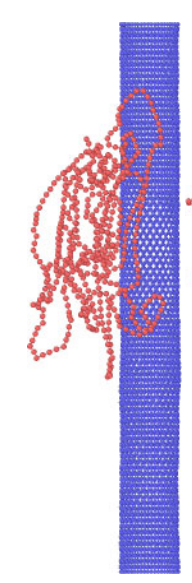

(a)

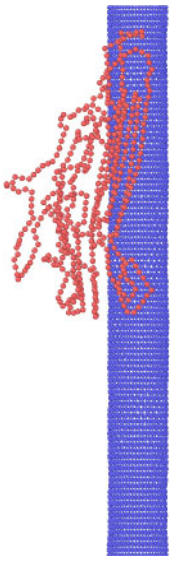

(b)

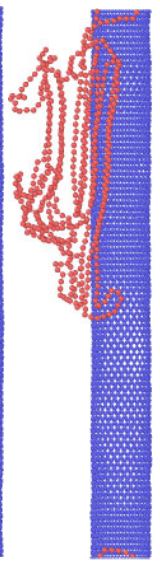

(c)

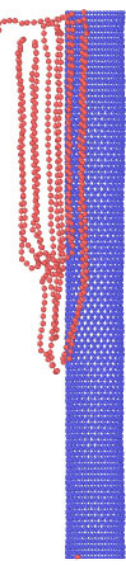

(d)

Fig. 5. Configuration change of a single chain during isothermal crystallisation. (a) time $=0.75 \mathrm{~ns}$, (b) time $=2.25 \mathrm{~ns},(\mathrm{c})$ time $=3.75 \mathrm{~ns}$ and $(\mathrm{d})$ time $=15 \mathrm{~ns}$

As the isothermal crystallisation process of polymer crystallisation on CNT surface is clear, non-isothermal crystallisation at a constant cooling rate, like a normal DSC experiment, was simulated for determination of the crystallisation temperature for the crystallisation on CNT surface and in the polymer matrix. Fig. 6 shows the enthalpy against temperature during the non-isothermal cooling test. Results indicated that there is an increase in crystallisation temperature in the CNT system, which agrees with the results of isothermal crystallisation. Ideally, the result should show two crystallisation peaks, one from the crystallisation on the surface of the CNT, and another one from that in the polymer matrix. However, due to the limitation of the computation capability, the cooling rate is too fast to distinguish the peaks between the two kinds of crystallisation. 


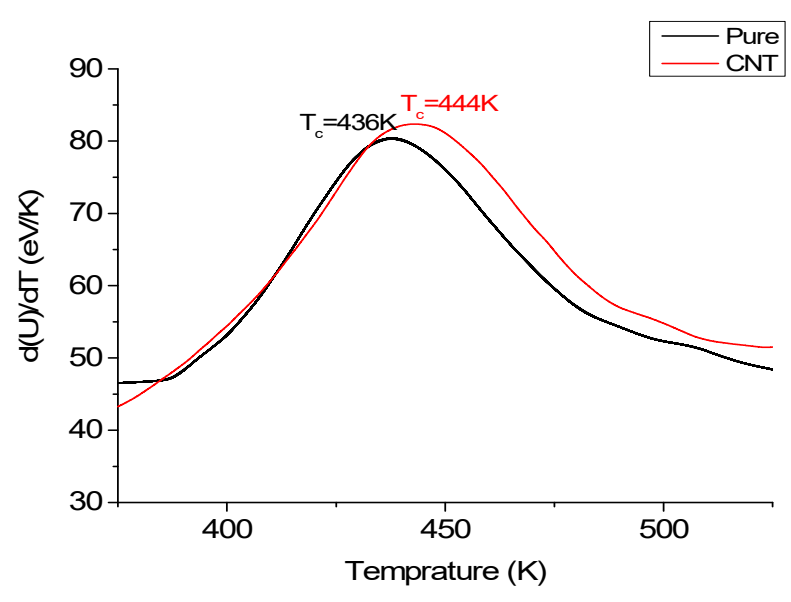

Fig. 6. Enthalpy against temperature during non-isothermal cooling test

The simulation results can be validated by the experimental data reported by Coleman et al [1], Lin et al. [33] and Acierno et al.[47].

To understand the effect of the crystallised layer formed on the surface of the CNT on the mechanical property, tensile test was carried out for the short fibre system in longitudinal and vertical directions of the CNT and for long fibre system in longitudinal direction for both amorphous and interface crystallised systems. Bulk crystallisation was avoided during those tests by controlling the crystallisation temperature at $440 \mathrm{~K}$ during isothermal crystallisation (which is significant faster $450 \mathrm{~K}$ ) for $15 \mathrm{~ns}$ and suddenly quench to $330 \mathrm{~K}$. The strain-stress curves are shown in Fig. 7. It was suggested experimentally [1] that a thicker polymer crystal layer can help the load transfer through polymer to CNT, caused bulk fracture in PVA/CNT composite, without any chemical bonding. 


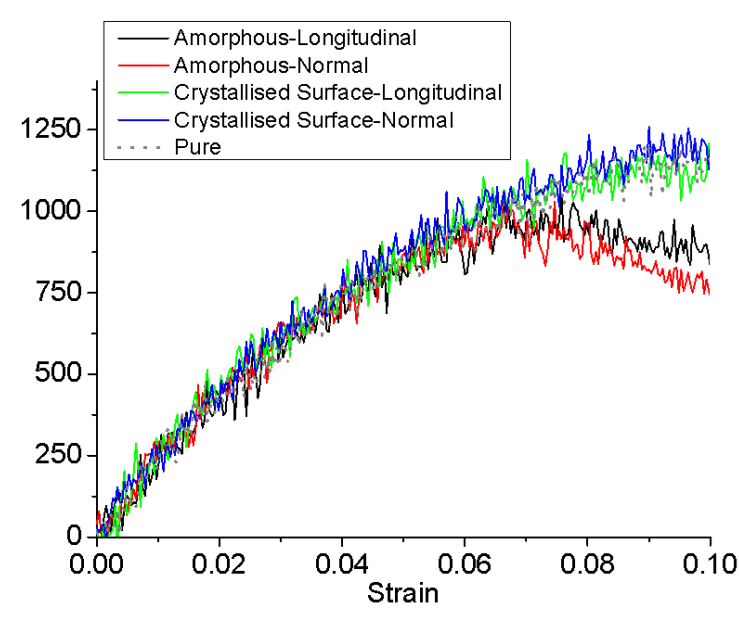

(a)

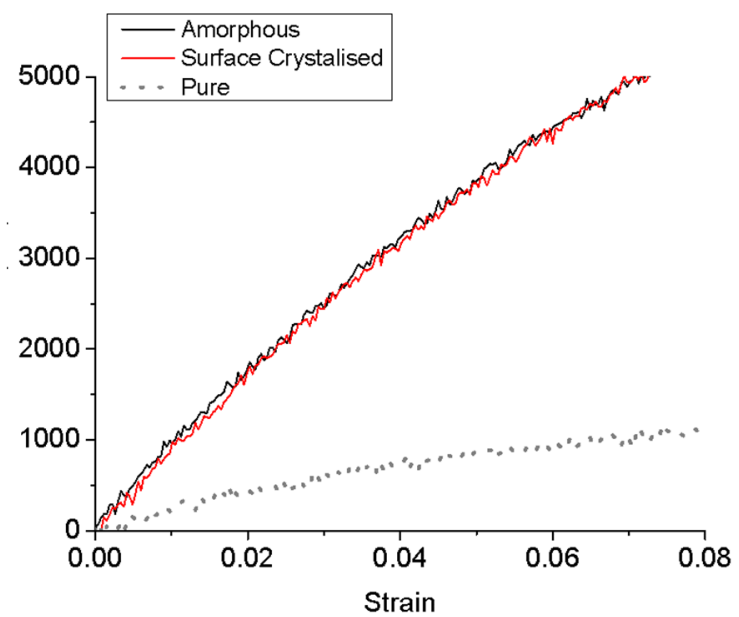

(b)

Fig. 7. Stress-Strain curve of (a) short CNT fibre and (b) continuous CNT fibre

For the short fibre system, the results shown in Fig. 7(a) indicate there is little difference at the initial stage between the surface crystallised composite or amorphous composite and the pure polymer. The fibre is too short to fully transfer the load. However, an early fracture at approx. strain $=0.07$ for amorphous systems was found. This early fracture indicates the CNT lowers the mechanical properties of system, as make it became more brittle with their limited length, which is significantly lower than the critical length. As CNT is much stiffer and stronger than the polymer, the crack happens at the interphase. This reveals that CNT-polymer interaction is not strong enough. On the other hand, the early fracture did not happen in the surface crystallised systems, indicating the formed crystals pause the interfacial failure. The stress-strain curves also showed little difference in the crystallised and amorphous continuous CNT fibre systems (Fig. 7(b)). Both are much stronger than the pure polymer system. As the stress was generated from iso-strain mode, the stress generated form the CNT extension in composite systems are much larger than other responses, i.e., extension, fracture of the polymer matrix and 
debonding. [48][49] The stress-strain behaviour in such a small system could not reveal the real situation. However, in the short fibre systems, the early fracture of the amorphous systems was not presented in the surface crystallised systems. To understand how the void concentrates and grows, snapshots of the simulation were used. The representative snapshots with polymer beads hidden for short fibre and long fibre scenarios are shown in Fig. 8. During tensile test, the voids were created and labelled in white colour shown in Fig. 8.
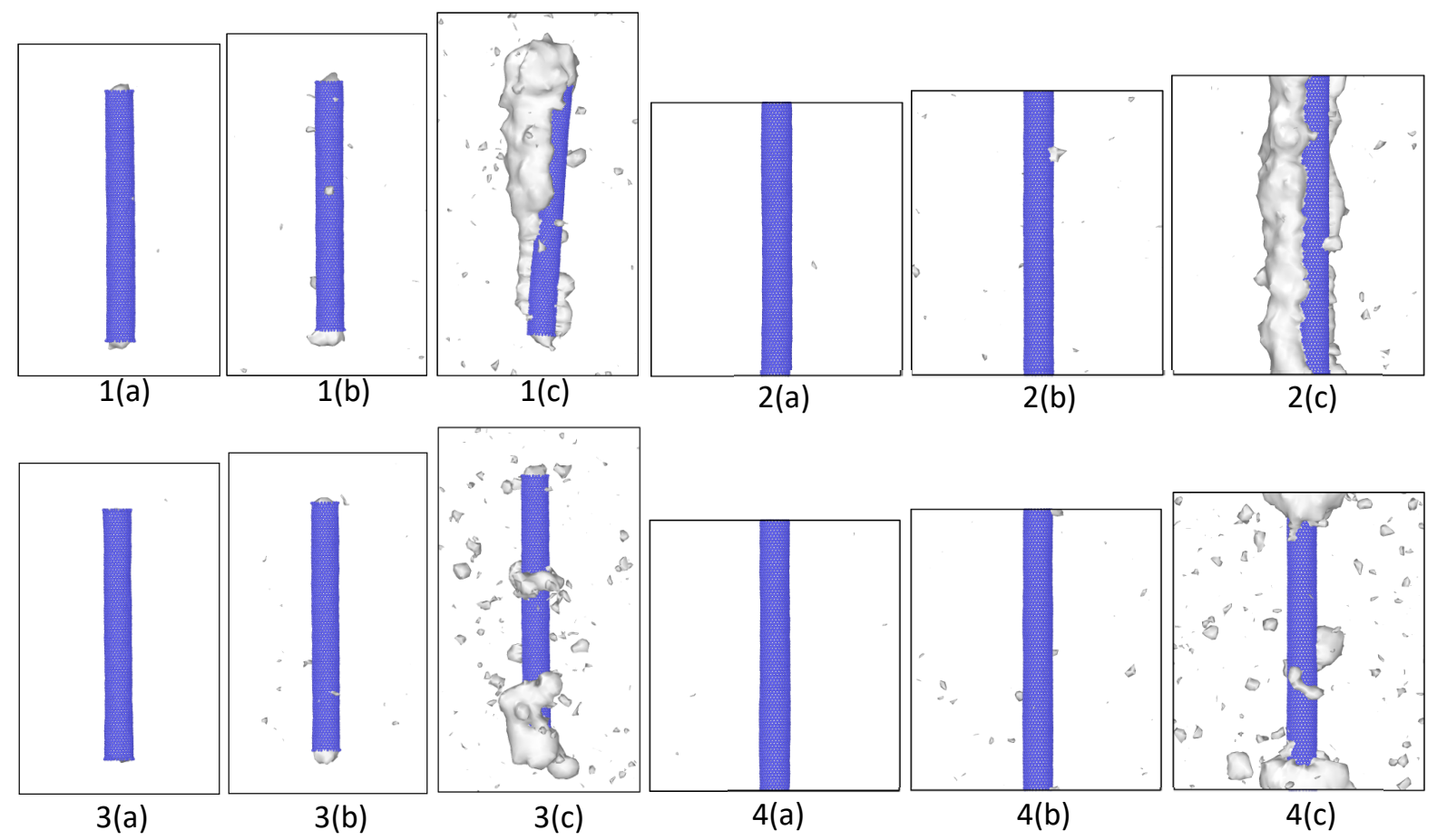

Fig. 8. Void propagation during tensile loading. (1) short CNT amorphous system, (2) long CNT amorphous system, (3) short CNT interfacial crystallised system and (4) long CNT interfacial crystallised system. (a) strain $=0$, (b) strain $=0.0375$ and (c) strain $=$ 0.09375

From Fig. 8-1 and Fig. 8-3, the void firstly generated at the end of the CNT for both 
amorphous and interphase crystallised systems, the same as the pull-out like fracture. Some small voids start to grow in the CNT-polymer interphase. In the amorphous system, the void growth is quite quick through the longitudinal direction of the CNT. The amorphous system failed at strain $\approx 0.07$. Fig. $8-1(\mathrm{c})$ shows a post-failure structure was formed in the amorphous system. The whole CNT-polymer interphase cracked, and CNT no longer support the polymer matrix leading to interphase completely failed. In the interphase crystallised system (Fig.8-3), the void growth is relatively slow, and cracks were more dis-continuous on the end of CNT compared with the amorphous system. As the crack is more dis-continuous, the CNT was still partially embedded in the polymer and the load can still be transferred. The crystals "paused" the growth of the cracks along the longitudinal direction of CNT resulting in lower void concentration.

Similar results were found in the snapshots of continuous CNT scenarios (Fig. 8-2 and Fig. 8-4). Because the CNT generates most of the stress during elongation, it was impossible to identify a failure point from stress-strain curve for neither the matrix nor interface. However, even more continuous fracture could be obtained from Fig. 8-2(c), which shows the crack quickly grows through the CNT surface longitudinally. Less CNT was embedded in the polymer matrix as cracks grow which will lower the efficiency of the load transfer between CNT and the polymer matrix. The crack growth in the surface crystallised systems was scattered and rather than formed a continue phase in the CNT-polymer interphase. In this case, the interphase was stronger and can transfer more load than in the amorphous systems. Because the CNT length is unlimited 
in the long fibre system, the pull-out like fracture could not be observed as there is no "end" of the CNT.

It is worth to mention that there are more voids in the matrix for the surface crystallised system than in the amorphous system for both short fibre and continuous fibre scenario. The results indicated failure of the amorphous system mainly occurs in the CNTpolymer interphase, and much more matrix failure happened in the surface crystallised system.

So far, how to understand the stress transfer in CNT/polymer nanocomposites from computer simulation is not clear. We attempt to develop a method for solving the problem. As the boundary effect was eliminated in the continuous fibre composite system, average surface energy was calculated. The surface energy at strain $=0$ was evaluated by calculating sum of the total energy contributed by interactions between the CNT and polymer chains. Table 1 shows the surface energy for the crystallised and amorphous systems.

Table 1 Surface energy of crystallised and amorphous systems

\begin{tabular}{|c|c|}
\hline System & $\sum E_{\varepsilon=0}(\mathrm{eV})$ \\
\hline Amorphous interphase & -34.87 \\
\hline Crystallised interphase & -40.12 \\
\hline
\end{tabular}

A lower surface energy was formed in the interface crystallised system. As the lower 
the surface energy, the more work will be required to de-bond the particular interphase, this is a more directly evidence that the crystallisation strengths the interaction between the CNT and polymer chains.

In the tensile test, if the load was not transferred through the interphase, the energy should be dispersed in the interphase to form the cavity in the interphase. So, by calculate the loss of the energy in the interphase, the load transfer could be assessed. The concept of percentage of lost surface energy against strain was applied, defined as follows.

Percentage of lost surface energy $(\operatorname{LSE} \%)=\frac{\sum E_{\varepsilon}-\sum E_{\varepsilon=0}}{\sum E_{\varepsilon=0}}$

The deviation of the LSE against strain $\left(\frac{d(L S E)}{d \varepsilon}\right)$ was used to characterise how fast the surface energy lost and how fast the interface decomposition is. The higher the value, the faster the interfacial energy drops which indicate the stress works instead transferred, and the faster the interfacial damage is.

The LSE-strain plots of amorphous and crystallised systems with different interaction strengths are shown in Fig. 9. 


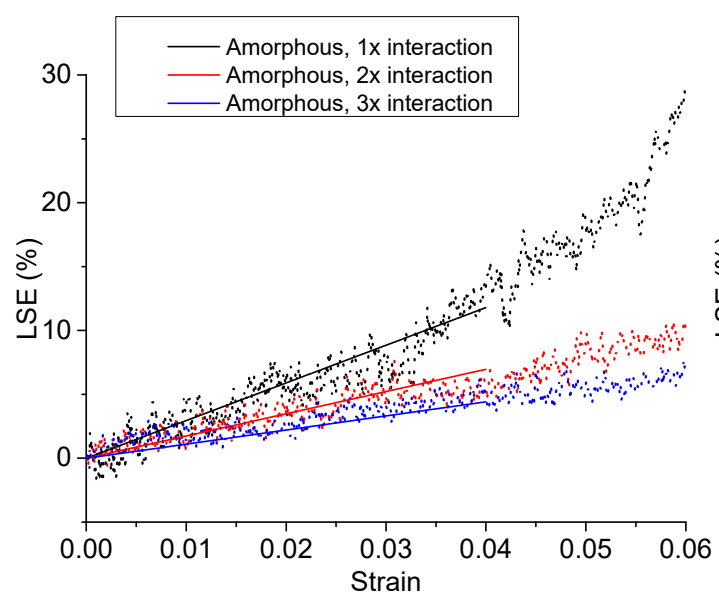

(a)

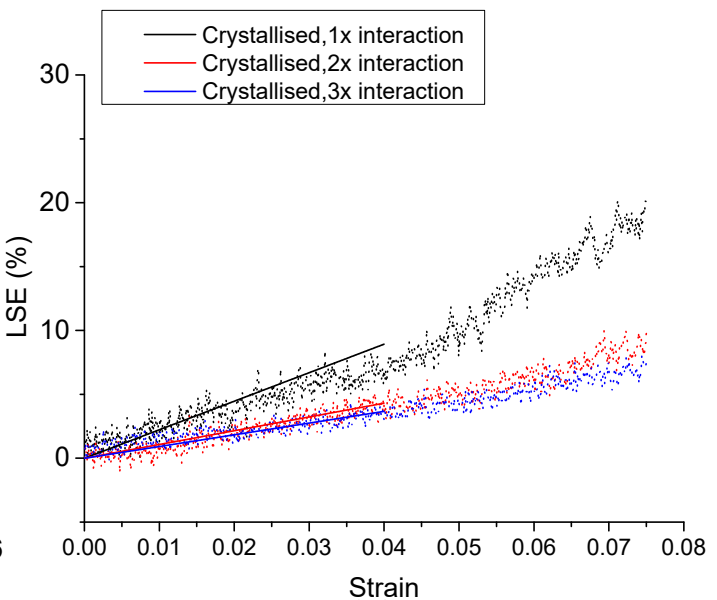

(b)

Fig. 9. LSE against strain with different interaction strengths. (a) Interfacial amorphous systems, and (b) interfacial crystallised systems. Different strength of CNT/CGPVA interaction potential were used by controlling the depth of the potential.

In general, a linear relationship can be found at the beginning of the strain $(\varepsilon<0.2)$. It is well-known if the strength of polymer-CNT interaction is increased, better stress transfer from the polymer matrix to CNT can be achieved. From Fig. 9(a), it is clear, with increasing the strength of polymer-CNT interaction, $\frac{d(L S E)}{d \varepsilon}$ will decreases, representing better stress transfer. $\frac{d(L S E)}{d \varepsilon}$ values for different systems are shown in Table 2.

Table $2 \frac{d(L S E)}{d \varepsilon}$ for different systems

\begin{tabular}{|l|l|l|}
\hline$\frac{d(L S E)}{d \varepsilon}$ & Amorphous & Crystallised \\
\hline $1 \times$ interaction & 2.95 & 2.23 \\
\hline $2 \times$ interaction & 1.74 & 1.07 \\
\hline $3 \times$ interaction & 1.10 & 0.92 \\
\hline
\end{tabular}


Comparing with the different systems which have different strengths of interaction, a descending trend can be found for the amorphous and crystallised systems. The change of the $\frac{d(L S E)}{d \varepsilon}$ with different strengths of interaction confirmed that the stronger the interaction (which leads to a better the stress transfer), the lower the $\frac{d(L S E)}{d \varepsilon}$.

The value of $\frac{d(L S E)}{d \varepsilon}$ in the initial part can be used to evaluate the interfacial failure rate. However, several factors will affect the interfacial debonding, such as chemical structure, crystal lamella structure and the thickness of the crystallised layer. These will be investigated in future work.

Thus, the analysis based on the change of $\frac{d(L S E)}{d \varepsilon}$ can be used to compare the amorphous and crystallised systems. For each particular strength of polymer-CNT interaction, the interfacial crystallised system has a smaller value of $\frac{d(L S E)}{d \varepsilon}$ than that of the amorphous system. Thus, a slower debonding occurred in the interfacial crystalized system when a tensile stress was applied. The interfacial crystallised layer can enhance the stress transfer in polymer/CNT nanocomposites and reduce the mismatch between CNT and the polymer matrix.

\section{Conclusions}

In this work, based on the molecular dynamics simulation, the crystallisation process, failure behaviour and stress transfer in poly (vinyl alcohol)/CNT nanocomposites were investigated. CNT induced crystallisation process can be divided into three stages of 
chain folding, orientating and growing on the CNT surface. The polymer chains first folded on CNT then orientated align with CNT and finally grew. An enhancement of mechanical behaviour was observed with the present of the polymer/CNT interfacial polymeric crystals. A slower crack growth was observed in the interfacial crystallised polymer/CNT systems, compare to relative amorphous systems. Better stress transfer was found with the polymer/CNT interfacial crystallisation, proved by the rate of interfacial debonding (characterised by $(\mathrm{d}(\mathrm{LSE})) / \mathrm{d} \varepsilon$ ). The enhancement on polymer/CNT interfacial bonding by formation of interfacial polymeric crystals was partially equivalent to the chemical modification of CNT. The interfacial interaction was critical to the final properties of a polymer/CNT nanocomposite. In fact, a short CNT fibre with a poor surface adhesion/interaction might lead to a detrimental result. Rather than commonly used chemical approach to enhance the interfacial bonding, this work is evident that it is possible to improve the CNT/polymer interfacial bonding by temperature control carefully during production of a semi-crystallised thermoplastic polymer/CNT nanocomposite as the polymer/CNT interfacial crystallisation takes place at a slightly higher temperature than at that of the bulk crystallization.

\section{Conflicts of interest}

There are no conflicts to declare.

\section{Acknowledgements}

Authors thank SABIC and EPSRC for providing funding for this work.

\section{Reference}

[1] J.N. Coleman, M. Cadek, R. Blake, V. Nicolosi, K.P. Ryan, C. Belton, A. 
Fonseca, J.B. Nagy, Y.K. Gun'ko, W.J. Blau, High-performance nanotubereinforced plastics: Understanding the mechanism of strength increase, Adv. Funct. Mater. 14 (2004) 791-798. doi:10.1002/adfm.200305200.

[2] H. Xia, M. Song, Preparation and characterization of polyurethane-carbon nanotube composites, Soft Matter. 1 (2005) 386. doi:10.1039/b509038e.

[3] J.K.W. Sandler, J.E. Kirk, I.A. Kinloch, M.S.P. Shaffer, A.H. Windle, Ultralow electrical percolation threshold in carbon-nanotube-epoxy composites, Polymer (Guildf). 44 (2003) 5893-5899. doi:10.1016/S0032-3861(03)00539-1.

[4] A. Wall, J.N. Coleman, M.S. Ferreira, Physical mechanism for the mechanical reinforcement in nanotube-polymer composite materials, Phys. Rev. B Condens. Matter Mater. Phys. 71 (2005) 1-5.

doi:10.1103/PhysRevB.71.125421.

[5] R.S. Lee, W.H. Chen, J.H. Lin, Polymer-grafted multi-walled carbon nanotubes through surface-initiated ring-opening polymerization and click reaction, Polymer (Guildf). 52 (2011) 2180-2188. doi:10.1016/j.polymer.2011.03.020.

[6] V.M. Martinez-Hernandez, Ana L.; Velasco-Santos, Carlos; Castano, Carbon Nanotubes Composites: Processing, Grafting and Mechanical and Thermal Properties, Curr. Nanosci. 6 (2010) 12-39. doi:10.2174/157341310790226270.

[7] N. Feng, X. Wang, D. Wu, Surface modification of recycled carbon fiber and its reinforcement effect on nylon 6 composites: Mechanical properties, morphology and crystallization behaviors, Curr. Appl. Phys. 13 (2014) 20382050. doi:10.1016/j.cap.2013.09.009.

[8] G.L. Hwang, Y.T. Shieh, K.C. Hwang, Efficient load transfer to polymergrafted multiwalled carbon nanotubes in polymer composites, Adv. Funct. Mater. 14 (2004) 487-491. doi:10.1002/adfm.200305382.

[9] M. Cadek, J.N. Coleman, V. Barron, K. Hedicke, W.J. Blau, Morphological and mechanical properties of carbon-nanotube-reinforced semicrystalline and amorphous polymer composites, Appl. Phys. Lett. 81 (2002) 5123-5125. doi:10.1063/1.1533118.

[10] M.L. Minus, H.G. Chae, S. Kumar, Observations on Solution Crystallization of Poly ( vinyl alcohol ) in the Presence of Single-Wall Carbon Nanotubes a, Macromol. Rapid Commun. 31 (2010) 310-316. doi:10.1002/marc.200900539.

[11] K.P. Ryan, M. Cadek, V. Nicolosi, S. Walker, M. Ruether, A. Fonseca, J.B. Nagy, W.J. Blau, J.N. Coleman, Multiwalled carbon nanotube nucleated crystallization and reinforcement in poly (vinyl alcohol) composites, Synth. Met. 156. 156 (2006) 332-335. doi:10.1016/j.synthmet.2005.12.015.

[12] M. Cadek, J.N. Coleman, K.P. Ryan, V. Nicolosi, G. Bister, A. Fonseca, J.B. Nagy, K. Szostak, F. Béguin, W.J. Blau, Reinforcement of Polymers with Carbon Nanotubes: The Role of Nanotube Surface Area, Nano Lett. 4 (2004) 353-356. doi:10.1021/n1035009o.

[13] M.L. Minus, H.G. Chae, S. Kumar, Single wall carbon nanotube templated oriented crystallization of poly(vinyl alcohol), Polymer (Guildf). 47 (2006) 3705-3710. doi:10.1016/j.polymer.2006.03.076.

[14] M. Hegde, E.T. Samulski, M. Rubinstein, T.J. Dingemans, The role of 
crystallinity in SWCNT - polyetherimide nanocomposites, Compos. Sci. Technol. 110 (2015) 176-187. doi:10.1016/j.compscitech.2015.02.004.

[15] X. Yan, Y. Imai, D. Shimamoto, Y. Hotta, Relationship study between crystal structure and thermal / mechanical properties of polyamide 6 reinforced and unreinforced by carbon fiber from macro and local view, Polymer (Guildf). 55 (2014) 6186-6194. doi:10.1016/j.polymer.2014.09.052.

[16] M.L. Minus, H.G. Chae, S. Kumar, Polyethylene Crystallization Nucleated by Carbon Nanotubes under Shear, ACS Appl. Mater. Interfaces. 4 (2012) 327330. doi:10.1021/am2013757.

[17] M. Nie, D.M. Kalyon, F.T. Fisher, Interfacial load transfer in polymer/carbon nanotube nanocomposites with a nanohybrid shish kebab modification, ACS Appl. Mater. Interfaces. 6 (2014) 14886-14893. doi:10.1021/am501879q.

[18] L. Li, C.Y. Li, C. Ni, Polymer crystallization-driven, periodic patterning on carbon nanotubes, J. Am. Chem. Soc. 128 (2006) 1692-1699. doi:10.1021/ja056923h.

[19] D. Kobayashi, A. Takahara, Interphase crystal structure of polyamide 6 on carbon materials revealed by grazing incidence X-ray diffraction with synchrotron radiation, Polymer (Guildf). 97 (2016) 174-178. doi:10.1016/j.polymer.2016.05.028.

[20] F. Zhang, H. Zhang, Z. Zhang, Z. Chen, Q. Xu, Modification of carbon nanotubes: Water-soluble polymers nanocrystal wrapping to periodic patterning with assistance of supercritical CO2, Macromolecules. 41 (2008) 4519-4523. doi:10.1021/ma800514a.

[21] B. Ni, S.B. Sinnott, P.T. Mikulski, J.A. Harrison, Compression of Carbon Nanotubes Filled with C60, CH4 or Ne: Predictions from Molecular Dynamics Simulations, Phys. Rev. Lett. 88 (2002) 205505. doi:10.1103/PhysRevLett.88.205505.

[22] Z.-Y. Ong, E. Pop, Molecular dynamics simulation of thermal boundary conductance between carbon nanotubes and SiO2, Phys. Rev. B. 81 (2010) 155408. doi:10.1103/PhysRevB.81.155408.

[23] V. Bala, S.K. Tripathi, R. Kumar, DFT study of CdS-PVA film, IOP Conf. Ser. Mater. Sci. Eng. 73 (2015) 012118. doi:10.1088/1757-899X/73/1/012118.

[24] H.L. Chou, C.M. Wu, F.D. Lin, J. Rick, Interactions between silver nanoparticles and polyvinyl alcohol nanofibers, AIP Adv. 4 (2014). doi:10.1063/1.4890290.

[25] O.C. Compton, S.W. Cranford, K.W. Putz, Z. An, L.C. Brinson, M.J. Buehler, S.T. Nguyen, Tuning the mechanical properties of graphene oxide paper and its associated polymer nanocomposites by controlling cooperative intersheet hydrogen bonding, ACS Nano. 6 (2012) 2008-2019. doi:10.1021/nn202928w.

[26] A. Noorjahan, P. Choi, Thermodynamic properties of poly(vinyl alcohol) with different tacticities estimated from molecular dynamics simulation, Polymer (Guildf). 54 (2013) 4212-4219. doi:10.1016/j.polymer.2013.05.073.

[27] D. Reith, H. Meyer, F. Müller-Plathe, Mapping atomistic to coarse-grained polymer models using automatic simplex optimization to fit structural 
properties, Macromolecules. 34 (2001) 2335-2345. doi:10.1021/ma001499k.

[28] F. Müller-Plathe, Coarse-graining in polymer simulation: From the atomistic to the mesoscopic scale and back, ChemPhysChem. 3 (2002) 754-769.

doi:10.1002/1439-7641(20020916)3:9<754::AID-CPHC754>3.0.CO;2-U.

[29] C. Luo, J.U. Sommer, Coding coarse grained polymer model for LAMMPS and its application to polymer crystallization, Comput. Phys. Commun. 180 (2009) 1382-1391. doi:10.1016/j.cpc.2009.01.028.

[30] T. Vettorel, H. Meyer, Coarse graining of short polythylene chains for studying polymer crystallization, J. Chem. Theory Comput. 2 (2006) 616-629. doi:10.1021/ct0503264.

[31] V.A. Harmandaris, N.P. Adhikari, N.F.A. Van Der Vegt, K. Kremer, Hierarchical modeling of polystyrene: From atomistic to coarse-grained simulations, Macromolecules. 39 (2006) 6708-6719. doi:10.1021/ma0606399.

[32] H.J. Qian, P. Carbone, C. Xiaoyu, H.A. Karimi-Varzaneh, C.C. Liew, F. Müller-Plathe, Temperature-Transferable Coarse-Grained potentials for ethylbenzene, polystyrene, and their mixtures, Macromolecules. 41 (2008) 9919-9929. doi:10.1021/ma801910r.

[33] J.-H. Lin, Z.-I. Lin, Y.-J. Pan, C.-T. Hsieh, C.-L. Huang, C.-W. Lou, Thermoplastic polyvinyl alcohol/multiwalled carbon nanotube composites: Preparation, mechanical properties, thermal properties, and electromagnetic shielding effectiveness, J. Appl. Polym. Sci. 133 (2016) n/a-n/a. doi:10.1002/app.43474.

[34] S.J. V Frankland, A. Caglar, D.W. Brenner, M. Griebel, Molecular Simulation of the Influence of Chemical Cross-Links on the Shear Strength of Carbon Nanotube - Polymer Interfaces, (2002) 3046-3048.

[35] Y. Zhang, X. Zhuang, J. Muthu, T. Mabrouki, M. Fontaine, Y. Gong, T. Rabczuk, Load transfer of graphene/carbon nanotube/polyethylene hybrid nanocomposite by molecular dynamics simulation, Compos. Part B Eng. 63 (2014) 27-33. doi:10.1016/j.compositesb.2014.03.009.

[36] M. Čanadija, R. Barretta, F.M. de Sciarra, On functionally graded Timoshenko nonisothermal nanobeams, Compos. Struct. 135 (2016) 286-296. doi:10.1016/j.compstruct.2015.09.030.

[37] R. Barretta, S. Ali Faghidian, R. Luciano, C.M. Medaglia, R. Penna, Stressdriven two-phase integral elasticity for torsion of nano-beams, Compos. Part B Eng. 145 (2018) 62-69. doi:10.1016/j.compositesb.2018.02.020.

[38] R. Barretta, S.A. Faghidian, R. Luciano, C.M. Medaglia, R. Penna, Free vibrations of FG elastic Timoshenko nano-beams by strain gradient and stressdriven nonlocal models, Compos. Part B Eng. 154 (2018) 20-32. doi:10.1016/j.compositesb.2018.07.036.

[39] R. Barretta, M. Čanadija, L. Feo, R. Luciano, F. Marotti de Sciarra, R. Penna, Exact solutions of inflected functionally graded nano-beams in integral elasticity, Compos. Part B Eng. 142 (2018) 273-286. doi:10.1016/j.compositesb.2017.12.022.

[40] R. Barretta, M. Diaco, L. Feo, R. Luciano, F.M. de Sciarra, R. Penna, Stress- 
driven integral elastic theory for torsion of nano-beams, Mech. Res. Commun. 87 (2017) 35-41. doi:10.1016/j.mechrescom.2017.11.004.

[41] M. Canadija, R. Barretta, F. Marotti De Sciarra, A gradient elasticity model of Bernoulli-Euler nanobeams in non-isothermal environments, Eur. J. Mech. A/Solids. 55 (2016) 243-255. doi:10.1016/j.euromechsol.2015.09.008.

[42] E. García-Macías, C.F. Guzmán, E.I. Saavedra Flores, R. Castro-Triguero, Multiscale modeling of the elastic moduli of CNT-reinforced polymers and fitting of efficiency parameters for the use of the extended rule-of-mixtures, Compos. Part B Eng. 159 (2019) 114-131. doi:10.1016/j.compositesb.2018.09.057.

[43] C. Luo, J.U. Sommer, Growth pathway and precursor states in single lamellar crystallization: MD simulations, Macromolecules. 44 (2011) 1523-1529. doi:10.1021/ma102380m.

[44] S. Plimpton, Fast Parallel Algorithms for Short - Range Molecular Dynamics, 117 (1995) 1-42.

[45] K.C. Alexander, C.A. Schuh, Visualization and analysis of atomistic simulation data with OVITO - the Open Visualization Tool, (n.d.). doi:10.1088/09650393/18/1/015012.

[46] W.G. Hoover, B.L. Holian, Kinetic moments method for the canonical ensemble distribution, Phys. Lett. Sect. A Gen. At. Solid State Phys. 211 (1996) 253-257. doi:10.1016/0375-9601(95)00973-6.

[47] S. Acierno, R. Barretta, R. Luciano, F. Marotti de Sciarra, P. Russo, Experimental evaluations and modeling of the tensile behavior of polypropylene/single-walled carbon nanotubes fibers, Compos. Struct. 174 (2017) 12-18. doi:10.1016/j.compstruct.2017.04.049.

[48] B. Arash, Q. Wang, V.K. Varadan, Mechanical properties of carbon nanotube/polymer composites, Sci. Rep. 4 (2014) 6479. doi:10.1038/srep06479.

[49] V. V. Mokashi, D. Qian, Y. Liu, A study on the tensile response and fracture in carbon nanotube-based composites using molecular mechanics, Compos. Sci. Technol. 67 (2007) 530-540. doi:10.1016/j.compscitech.2006.08.014. 